\section{Asociación entre enfermedad de von Willebrand y angiodisplasia: ¿casualidad o causalidad?}

\author{
JOSÉ SALINAS LAVAL ${ }^{1}$, NICOLÁS TRIANTAFILO², PAMELA ZÚÑIGA ${ }^{3}$
}

\section{Association between von Willebrand disease and angiodysplasia}

Von Willebrand factor ( $v W f$ ) is a fundamental multimeric plasma glycoprotein in the coagulation process. Its function is to mediate platelet adhesion and to stabilize circulating factor VIII. A functional or quantitative alteration of $v W f$ gives rise to von Willebrand disease ( $v W D)$. The association between $v W D$ and angiodysplasia was described in 1967, but it was only until 2011 that Starke et al demonstrated the in vitro and in vivo role of $v W f$ in angiogenesis. Congenital or acquired $v W f$ deficiency, especially of high molecular weight multimeters, not only favors bleeding, but also contributes to increased angiogenesis in these patients. The treatment should be focused both on the control of the acute episode of gastrointestinal bleeding, with $v W$ f replacement therapy and local endoscopic treatment, as well as on the prevention of the progression of angiodysplasia and future bleeding. There are different published therapeutic approaches using $v W f$ replacement that are not effective in all patients. Recently, angiogenesis inhibitor medications have been used.

(Rev Med Chile 2020; 148: 1475-1480)

Key words: Angiodysplasia; Gastrointestinal Hemorrhage; von Willebrand Diseases.
'Programa de formación de subespecialidad en Hematología adultos, Facultad de Medicina, Universidad Austral de Chile. Valdivia, Chile.

2Programa de formación de subespecialidad en Hematología adultos, Facultad de Medicina, Pontificia Universidad Católica de Chile. Santiago, Chile.

${ }^{3}$ Departamento de Pediatría, Facultad de Medicina, Pontificia Universidad Católica de Chile. Santiago, Chile.

Trabajo no recibió financiamiento. Los autores declaran no tener conflictos de interés.

Recibido el 26 de agosto de 2019, aceptado el 20 de marzo de 2020.

Correspondencia a:

Pamela Zúñiga

Departamento de Pediatría, Facultad de Medicina, Pontificia Universidad Católica de Chile. Santiago, Chile. pzuniga@med.puc.cl
$\mathrm{E}$ 1 factor de von Willebrand (vWf) es una glicoproteína multimérica plasmática. Su función es mediar la adhesión plaquetaria vía unión a GP Ib-IX-V, además, estabiliza al factor VIII (FVIII) en circulación previniendo su proteólisis ${ }^{1}$. Su disfunción cuantitativa o cualitativa genera el trastorno hereditario de la coagulación más común, la enfermedad de von Willebrand (vWD).

El vWf es sintetizado en células endoteliales, megacariocitos y plaquetas, y es almacenado en cuerpos de Weibel-Palade (WPBs) y gránulos alfa plaquetarios, respectivamente. En el aparato de Golgi se forman multímeros y adquieren una conformación tubular en los WPBs, luego, al fusionarse con la membrana plasmática de las células endoteliales, estos multímetros ultralargos son clivados en su dominio A2 por la metaloproteasa ADAMTS13 a hebras más pequeñas que circulan en el plasma ${ }^{2}$.

EL vWf circulante al unirse el subendotelio, en caso de daño, se desenrolla lo que le permite interactuar con GP Ib-IX-V, dando lugar a la fase de adhesión, lo que genera, a su vez, la activación de GP IIb-IIIa, que es primordial para la agregación plaquetaria.

La enfermedad de von Willebrand refleja la disminución de la síntesis del factor, vWD tipo 1 y 3 , o su disfunción vWD tipo 2. Estas alteraciones pueden deberse a alelos nulos (vWD tipo 1 y 3 ), mutaciones missense en sus diferentes dominios (vWD tipo 1 y 2$)^{2}$ (Tabla 1 ).

La asociación entre vWD y angiodisplasia se reporta hasta en $20 \%$ de los pacientes, especialmente 
Tabla 1. Características de laboratorio de los diferentes tipos de vWD

\begin{tabular}{|c|c|c|c|c|c|c|}
\hline & Tipo 1 & Tipo 2A & Tipo 2 B & Tipo 2 M & Tipo 2N & Tipo 3 \\
\hline VWF:Ag & Disminuido & Disminuido & Disminuido & Disminuido & Normal & $<5$ ui/dl \\
\hline VWF:RCo & $<30$ ui/dl & $<30$ ui/dl & $<30$ ui/dl & $<30$ ui/dl & Normal & - \\
\hline $\begin{array}{l}\text { Prueba de unión } \\
\text { a colágeno }\end{array}$ & Disminuido & Disminuido & Disminuido & Disminuido & Normal & - \\
\hline FVIII:C & $\begin{array}{l}\text { Normal o } \\
\text { disminuido }\end{array}$ & $\begin{array}{l}\text { Normal o } \\
\text { disminuido }\end{array}$ & $\begin{array}{l}\text { Normal o } \\
\text { disminuido }\end{array}$ & $\begin{array}{l}\text { Normal o } \\
\text { disminuido }\end{array}$ & $\begin{array}{c}\text { Muy } \\
\text { disminuido }\end{array}$ & $\begin{array}{c}\text { Muy } \\
\text { disminuido }\end{array}$ \\
\hline Múltimeros & Normal & $\begin{array}{l}\text { Pérdida de } \\
\text { múltimeros de alto } \\
\text { peso molécular }\end{array}$ & $\begin{array}{l}\text { Pérdida de } \\
\text { múltimeros de alto } \\
\text { peso molécular }\end{array}$ & Normal & Normal & - \\
\hline VWF: FVIIIB & Normal & Normal & Normal & Normal & Disminuida & - \\
\hline $\begin{array}{l}\text { Recuento de } \\
\text { plaquetas }\end{array}$ & Normal & Normal & Bajo & Normal & Normal & Normal \\
\hline RIPA & Ausente & Ausente & $\begin{array}{l}\text { Muy aumentada, } \\
\text { incluso }<0,6 \mathrm{mg} / \mathrm{dl}\end{array}$ & Ausente & Ausente & Ausente \\
\hline
\end{tabular}

VWF:Ag: Factor von Willebrand antigénico; FVIII:C: Factor VIII coagulante; vWF:FVIIIB: Prueba de unión factor von Willebrand-factor VIII; RIPA: agregrometría con dosis crecientes de ristocetina.

tipo 2 A y 3 , donde se asocia a la pérdida de multímeros de alto peso molecular ${ }^{5}$. La angiodisplasia es más frecuente en el ciego y colon ascendente, pero se ha descrito en todo el tubo digestivo.

En 1967, Quick reportó pacientes con vWD y telangectasias nasales ${ }^{3}$. En 1976, se reportaron los primeros casos de angiodisplasia gastrointestinal ${ }^{23}$.

Finalmente, en el año 2011, se describió el papel del factor de von Willebrand como un mediador de la angiogénesis ${ }^{7}$.

El propósito de esta revisión es plantear la fisiopatología involucrada en esta asociación y describir opciones de terapia descritas en la literatura a la luz del papel del vWf en la angiogénesis.

\section{$\underline{\text { Fisiopatología }}$}

Las angiopoyetinas, angiopoyetina $-1 \mathrm{y}$ angiopoyetina -2 , actúan durante las fases finales de la angiogénesis. La angiopoyetina - 1 tiene una actividad mitogénica débil, causa la maduración de células endoteliales. La angiopoyetina -2 es el antagonista natural de angiopoyetina -1 en las células endoteliales, inhibe la maduración vascular, lo que permite mejor respuesta ante el estímulo del factor de crecimiento vascular endotelial (VEGF). Ambas angiopoyetinas se unen al mismo receptor
2 de cinasa de tirosina (Tie-2) específico de las células endoteliales y hematopoyéticas, el cual es activado por la angiopoyetina-1 y bloqueado por la angiopoyetina- 2 .

En condiciones normales, sin hipoxia, existe un ambiente que reprime la angiogénesis producto de la interacción de angiopoyetina - 1 con su receptor Tie-2. A esto se agrega la interacción de vWf con su receptor, la integrina avB3, lo cual inhibe la unión del factor de crecimiento vascular endotelial (VEGF) a su receptor (VEGFR-2) ${ }^{4,5}$.

Se ha reportado que $\mathrm{vWf}$ es primordial para la formación de cuerpos de Weibel-Palade (WPBs), y que estos contienen en su interior, además de vWf, proteínas de la matriz extracelular, interleuquinas, receptores de membrana, citoquinas y angiopoyetina $-2^{6}$.

Al haber disminución de la función o cantidad de vWf, no se formarían correctamente los WPBs, por lo cual, angiopoyetina -2 sería liberada sin mediar el control que ofrece WBPs, generando el aumento de su acción por medio de receptor Tie2 , inhibiendo competitivamente a angiopoyetina -1. Además, el vWF no se uniría a integrina avB3, lo cual generaría que el VEGF se uniera sin impedimento a su receptor (Figura 1).

Esta asociación causal fue demostrada por Starke y $\mathrm{col}^{7}$ en ratones knockout para vWf, en 
los cuales se observó un aumento marcado de la proliferación endotelial y un aumento de la neovascularización.

Este fenómeno también ha sido descrito en estenosis aórtica grave, en donde E.C. Heyde describe, en 1958, pacientes con la triada de estenosis aórtica, angiodisplasia y sangrado gastrointestinal, referido como el síndrome de Heyde ${ }^{8}$. El aumento del flujo en la válvula estenosada (shear stress) hace al vWF susceptible a ser clivado por ADAMTS13, resultando en la pérdida de multímeros de alto peso molecular.

Lo mismo ocurre en enfermedad de von Willebrand adquirida por generación de autoanticuerpos tipo IgG contra el complejo vWF-FVIII ${ }^{9}$, en enfermedades autoinmunes, trastornos linfoproliferativos crónicos, gammapatías monoclonales.

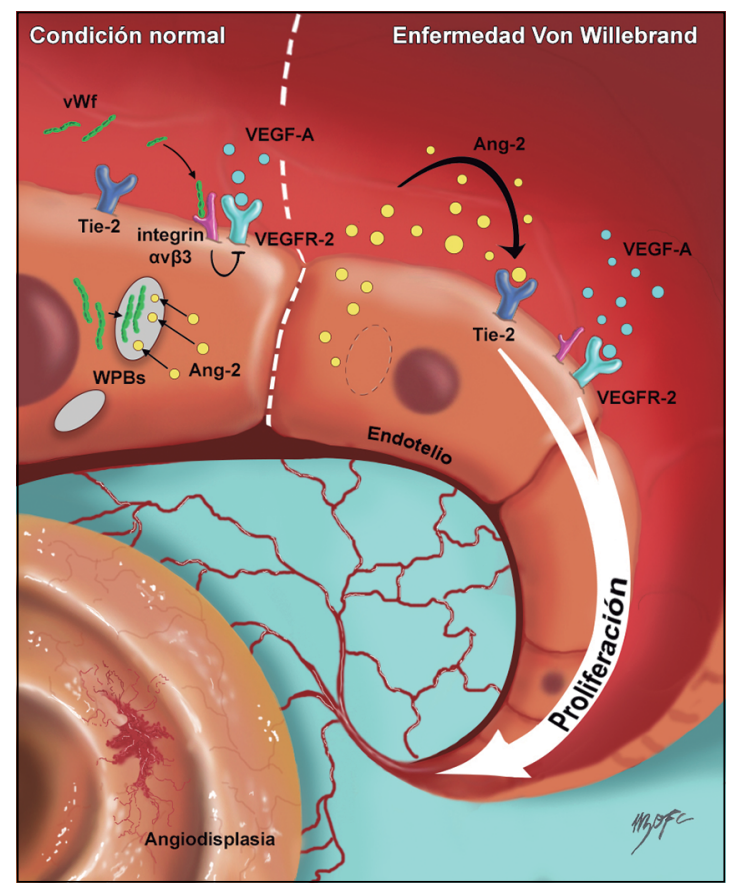

Figura 1. A la izquierda: en condiciones normales la presencia de factor de von Willebrand (vWf) permite, por un lado, la estabilidad de los cuerpos de Weibel-Palade (WPBs), capturando la angiopoyetina 2 (Ang-2) y por otro lado, la inhibición de la señalización intracelular del receptor VEGFR-2 por medio de la unión con la integrina av $\beta 3$. A la derecha: en enfermedad de von Willebrand (VWD), la deficiencia de vWf impide la formación de los WPBs, lo que produce la liberación de la Ang-2 que se une al receptor Tie-2. En conjunto con la ausencia de inhibición de VEGFR-2 por integrina av $\beta 3$ sin vWf, se promueve finalmente la proliferación endotelial.

\section{Enfrentamiento terapéutico del sangrado gastrointestinal por angiodisplasia}

El tratamiento en el episodio agudo se basa principalmente en el tratamiento endoscópico de la lesión sangrante. Esto puede ser complejo, ya que el sangrado puede no ser evidente, a veces intermitente, presentándose como anemia ferropriva ${ }^{10}$. Al mismo tiempo que el tratamiento local endoscópico, es necesario hacer una terapia de reemplazo, preferentemente con liofilizado de vWf (40-60 ui/ $/ \mathrm{kg} /$ día) o, en su ausencia, crioprecipitado.

La literatura sobre el tema y las guías clínicas de tratamiento de vWD concuerdan que, por su gran tendencia a recurrir, la hemorragia digestiva asociada a angiodisplasia, es una indicación de tratamiento profiláctico (vWf liofilizado dos o tres veces por semana). Esto fue evaluado en una publicación de la von Willebrand Disease Prophylaxis Network (vWD PN) en donde la recurrencia, a pesar de tratamiento profiláctico, fue más alta en pacientes con vWD y sangrado gastrointestinal que en pacientes con otros sangrados mucocutáneos ${ }^{11}$.

Ante la dificultad de tratamiento local, la alta tasa de recurrencia y el consto de mantener un tratamiento profiláctico en vWf se ha planteado el uso de diversos medicamentos con efecto antiangiogénico para abordar la terapia de pacientes con vWD y angiodisplasia intestinal. La Tabla 2 resume los reportes de casos publicados.

La talidomida ha sido uno de los medicamentos utilizados por su papel como modificador del control inmunológico (Imids) dada por su capacidad de inhibir el VEGF ${ }^{13}$.

La dosis de talidomida descrita es de $50 \mathrm{mg}$ vía oral al día, con aumento cada 4 semanas hasta dosis de $150 \mathrm{mg}$, se ha observado estabilización de la hemoglobinemia y disminución de los requerimientos de liofilizados en los 6 meses de seguimiento $^{14,15}$.

Hay una serie de casos de 5 pacientes con uso de lenalidomida ${ }^{21}$ en dosis inicial de $5 \mathrm{mg}$, hasta dosis de $15 \mathrm{mg}$ vía oral, que destaca ausencia de sangrado con una media de 12,6 meses y disminución del número de endoscopias $(\mathrm{p}=0,001)$. El seguimiento de estos pacientes fue por 2 años.

Otras publicaciones sugieren el uso de análogos de la somastostatina, como octreotide ${ }^{12,18}$, usado en dosis de 500 ug endovenosa por dos días y luego 


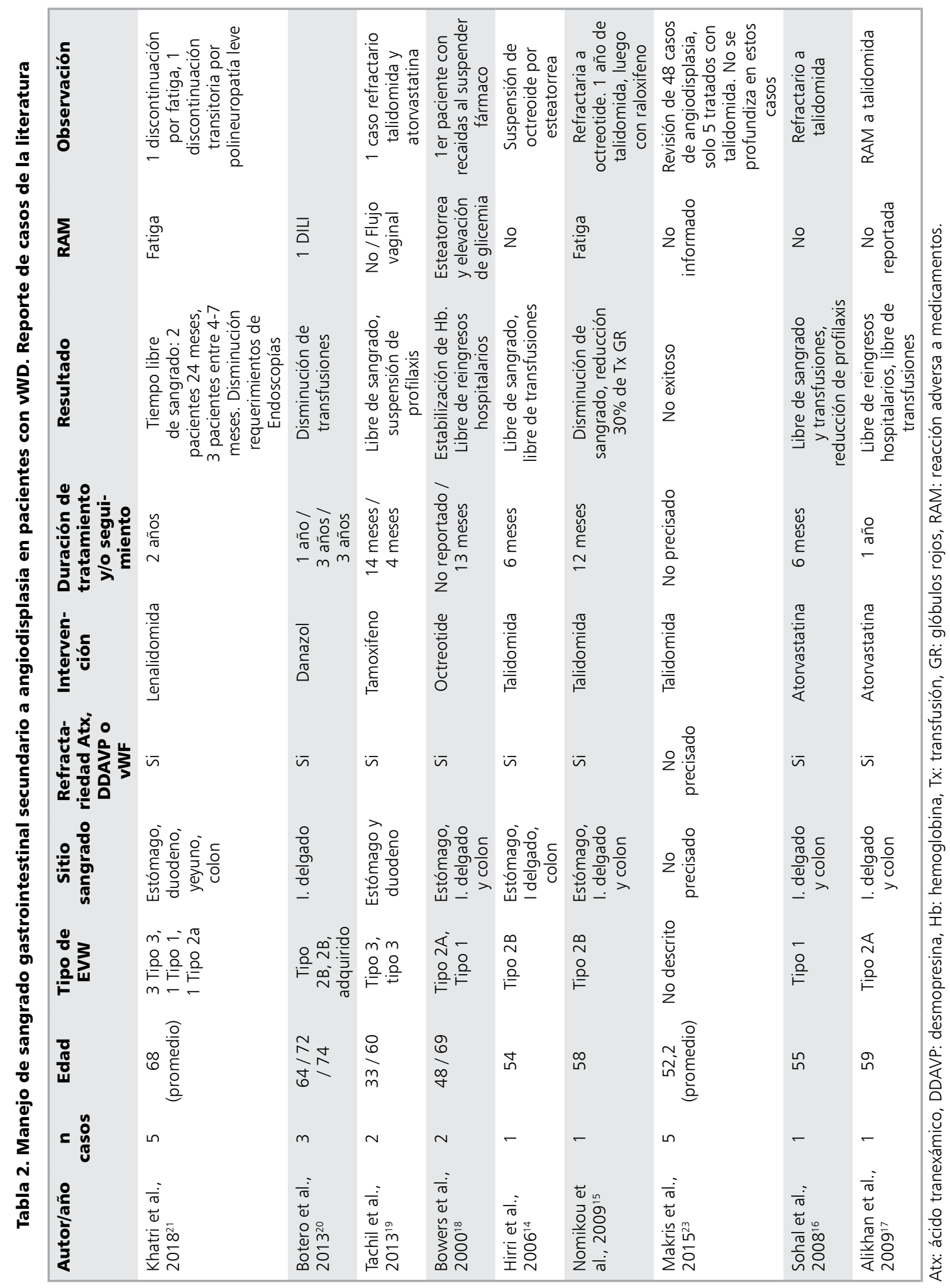


250 ug subcutáneo tres veces al día en el episodio de sangrado agudo, con una dosis de mantención de 100 ug subcutáneo en días alternos. En este caso, la paciente no requirió nuevos ingresos hospitalarios en un período de seguimiento de 13 meses.

La atorvastatina también se ha descrito como posible terapia, se describe un caso con dosis inicial de $10 \mathrm{mg}$ vo en la noche, aumentando $10 \mathrm{mg}$ por mes para hasta dosis de $80 \mathrm{mg}$, en este caso se observó disminución de hospitalizaciones y necesidad de transfusión de glóbulos rojos en un seguimiento de 9 meses $^{16}$.

Otro reporte del uso de atorvastatina, usando dosis escalonadas de $10 \mathrm{mg}$, llegando hasta $40 \mathrm{mg}$ en un período de 3 meses ${ }^{17}$ mostró una estabilización de la hemoglobinemia en un periodo de 6 meses, pero se mantuvo el uso de profilaxis con vWf, 1 vez a la semana.

Tamoxifeno ${ }^{19}$ se probó en un paciente varón que no había respondido a talidomida/atorvastatina. Su uso fue propuesto por su rol en el tratamiento de la telangectasia hereditaria hemorrágica, siendo su rol antriestrogénico el causante de su efecto antiangiogénico. Se observó una respuesta completa a los 3 meses, sin evidencia endoscópica de angiodisplasia, con discontinuación de la profilaxis con liofilizado de vWf.

Danazol $^{20}$ a dosis inicial de $100 \mathrm{mg}$ día, hasta dosis máxima de $500 \mathrm{mg}$ vo día, se reporta en tres pacientes, con beneficios similares a los descritos para otros medicamentos, pero en un caso el paciente presentó injuria hepática asociada a drogas (DILI).

\section{Discusión}

La finalidad de esta revisión fue revelar el rol del factor von Willebrand en el desarrollo de angiodisplasia gastrointestinal, desde una relación casual ya descrita en la década de 1960, hasta su confirmación causal descrita por Starke y $\mathrm{col}^{7} \mathrm{en}$ el 2011.

Al comprender la fisiopatología de esta asociación podemos plantear un enfoque terapéutico que comprenda, por una parte, el tratamiento del episodio de sangrado agudo $y$, por otra parte, el diseño de estrategias para prevenir recurrencias y evitar la progresión de la angiodisplasia.

En el tratamiento habitual de pacientes con
vWD y sangrado recurrente por angiodisplasia intestinal se describe, además del tratamiento local, la profilaxis con liofilizado de vWf, que consiste en aplicar liofilizado intravenoso 2 o 3 veces por semana.

Este tratamiento no ha demostrado ser tan eficiente como en otros sangrados mucocutáneos, es de alto costo y no está cubierto en Chile por las garantías explícitas en salud (GES), por lo que es de difícil acceso para muchos de nuestros pacientes.

Según lo revisado, pensamos que fundamentos fisiológicos para incluir dentro del algoritmo de manejo de estos pacientes el uso de inhibidores de la angiogénesis. Según la escasa bibliografía, su uso podría disminuir la tasa de sangrado recurrente, la necesidad de endoscopías terapéuticas y el uso de liofilizado de $\mathrm{vWf}^{12,14}$.

Cabe señalar que los reportes de casos pueden estar sujetos a sesgo de población $y$, por tanto, se necesitan estudios bien diseñados para evaluar la eficacia y los efectos secundarios de estos fármacos para la terapia de la hemorragia gastrointestinal en pacientes con $\mathrm{vWD}^{12}$.

Por ahora, y por lo poco frecuente de esta patología, no podemos dar una recomendación de tratamiento con alguno de estos medicamentos, posiblemente, la elección debiera basarse en la experiencia del manejo de las distintas terapias el acceso a ellas y los posibles efectos adversos, según cada paciente.

\section{Referencias}

1. Hoffman R, et al Editores. Hematology: Basic Principles and Practice. 8th edition. Philadelphia, PA: Elsevier, 2018.

2. Leebeek FWG, Eikenboom JCJ. Von Willebrand's Disease. N Engl J Med 2017; 376 (7): 701-2.

3. Selvam S, James P. Angiodysplasia in von Willebrand disease: understanding the clinical and basic science. Semin Thromb Hemost 2017; 43 (6): 572-80.

4. Franchini M, Mannucci PM. Gastrointestinal angiodysplasia and bleeding in von Willebrand disease. Thromb Haemost 2014; 112: 427-31.

5. Randi AM, Smith KE, Castaman G. von Willebrand factor regulation of blood vesselformation. Blood 2018; 132: 132-40.

6. Randi AM, Laffan MA, Starke RD. Von Willebrand factor, angiodysplasia and angiogenesis. Mediterr J Hematol Infect Dis 2013; 5 (1): e2013060. 
7. Starke RD, Ferraro F, Paschalaki KE, Dryden NH, McKinnon TA, Sutton RE, et al. Endothelial von Willebrand factor regulates angiogenesis. Blood 2011; 117: 1071-80.

8. Heyde EC. Gastrointestinal bleeding in aortic stenosis. N Engl J Med 1958; 259: 196.

9. Alhumood SA, Devine DV, Lawson L, Nantel SH, Carter CJ. Idiopathic immune-mediated acquired von Willebrand's disease in a patient with angiodysplasia: Demonstration of an unusual inhibitor causing a functional defect and rapid clearance of von Willebrand factor. Am J Hematol 1999; 60: 151-7.

10. Franchini M, Mannucci PM. Von Willebrand disease-associated angiodysplasia: a few answers, still many questions. Br J Haematol 2013; 161 (2): 177-82.

11. Abshire TC, Federici AB, Álvarez MT, Bowen J, Carcao $\mathrm{MD}$, Cox Gill J, et al. Prophylaxis in severe forms of von Willebrand's disease: results from the von Willebrand Disease Prophylaxis Network (vWD PN). Haemophilia 2013; 19 (1): 76-81.

12. LeebeekFWG, Atiq F. How to manage severe von Willebrand disease. Br. J Haematol 2019; 187(4): 418-30.

13. Bartlett JB, Dredge K, Dalgleish AG. The evolution of thalidomide and its IMiD derivatives as anticancer agents. Nat Rev Cancer 2004; 4 (4): 314-22.

14. Hirri HM, Green PJ, Lindsay J. Von Willebrand's disease and angiodysplasia treated with thalidomide. Haemophilia 2006; 12 (3): 285-6.

15. Nomikou E, Tsevrenis V, Gafou A, Bellia M, Theodossiades G. Type IIB Von Willebrand disease with angiodysplasias and refractory gastrointestinal bleeding successfully treated with thalidomide. Haemophilia 2009; 15 (6): 1340-2.

16. Sohal M, Laffan M. Von Willebrand disease and an- giodysplasia responding to atorvastatin. $\mathrm{Br} \mathrm{J}$ Haematol 2008; 142 (2): 308-9.

17. Alikhan R, Keeling D. Von Willebrand disease, angiodysplasia and atorvastatin. Br J Haematol 2010; 149 (1): 159-60.

18. Bowers M, McNulty O, Mayne E. Octreotide in the treatment of gastrointestinal bleeding caused by angiodysplasia in two patients with von Willebrand's disease. Br J Haematol 2000; 108 (3): 524-7.

19. Thachil J, Hay CR, Campbell S. Tamoxifen for recurrent bleeds due to angiodysplasia in von Willebrand's disease. Haemophilia 2013; 19 (5): e313-5.

20. Botero JP, Pruthi RK. Refractory bleeding from intestinal angiodysplasias successfully treated with danazol in three patients with von Willebrand disease. Blood Coagul Fibrinolysis 2013; 24 (8): 884-6.

21. Khatri NV, Patel B, Kohli DR, Solomon SS, Bull-Henry $\mathrm{K}$, Kessler CM. Lenalidomide as a novel therapy for gastrointestinal angiodysplasia in von Willebrand disease. Haemophilia 2018; 24 (2): 278-82.

22. Tsagianni A, Comer DM, Yabes JG, Ragni MV. Von Willebrand disease and gastrointestinal bleeding: A national inpatient sample study. Thromb Res 2019; 178: 119-23.

23. Makris M, Federici AB, Mannucci PM, Bolton-Maggs $\mathrm{PH}$, Yee TT, Abshire T, et al. The natural history of occult or angiodysplastic gastrointestinal bleeding in von Willebrand disease. Haemophilia 2015; 21 (3): 338-42.

24. Ramsay DM, Buist TAS, Macleod DAD, Heading RC. Persistent gastrointestinal bleeding due to angiodysplasia of the gut in von Willebrand's disease. Lancet 1976; 2 (7980): 275-8. 